\title{
Advances in pulmonary and pleural malignant disorders
}

\author{
Authors: Jack A Kastelik, ${ }^{A}$ Angshu Bhowmik ${ }^{B}$ and John Park ${ }^{C}$
}

Lung and pleural malignancies remain common in the UK with poor survival rates due, at least in part, to late stage diagnosis. Diagnostic pathways aim to reduce the time taken for patients to reach a diagnosis and treatment, with the use of positron emission tomography and endobronchial ultrasound to provide staging information alongside diagnostics. Advances in molecular phenotyping of tumours and the development of treatments to target these have provided new therapeutic options which can be individualised to patients. In the UK, screening for lung cancer remains in its infancy, but provides a promising possibility for capturing curative disease. We provide an overview of the diagnostic process, therapeutic options and potential future screening programmes in pleural and pulmonary malignancies.

\section{Introduction}

Pulmonary and pleural malignancies are common. Each year worldwide and in the UK approximately 1.8 million and 39,000 people were diagnosed with lung cancer and 1.6 million and 35,000 died from the disease, respectively. ${ }^{1}$ Similarly, mortality rates from malignant mesothelioma were reported at 4.9 per million, with a male to female ratio of 3.6:1. ${ }^{2}$ Many patients with lung cancer are diagnosed with late stage disease, which carries 5 -year survival of around $4 \%$, with only $5.5 \%$ of cases cured. ${ }^{1}$ Five-year survival rates for malignant mesothelioma are similarly poor, between $5 \%$ and $10 \%$, with median survival between 12 to 21 months for stage 4 and stage 1 disease, respectively. ${ }^{3}$ However, with the development of targeted therapy and immunotherapy it is likely that the survival will improve. Overall, around $90 \%$ of cases of lung cancer are caused by tobacco smoking. Asbestos exposure remains the most important contributing factor for malignant mesothelioma with a latency period of around 20 to 40 years to disease manifestation. Exposure can be occupational, domestic (eg household member exposure through the asbestos deposited on the work clothes) or environmental (eg living in the proximity of asbestos factories) and highlights the importance of a detailed history. ${ }^{3}$ World Health Organization (WHO) classification describes four histological

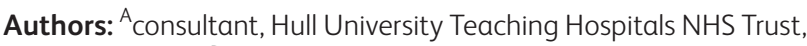
Cottingham, UK; ${ }^{B}$ consultant, Homerton University Hospital NHS Foundation Trust, London, UK; ${ }^{C}$ consultant, Royal Berkshire Hospital NHS Foundation Trust, Reading, UK types of mesothelioma including epithelioid (60\%), sarcomatoid (10-15\%), biphasic (25-30\%) and the rare desmoplastic. ${ }^{3}$ Recent Royal College of Physicians National Lung Cancer Audit report suggests that $10 \%$ of lung cancers are of small cell subtype (SCLC), carrying a worse prognosis. $88.5 \%$ are non-small cell subtype (NSCLC), which can be further subdivided into adenocarcinoma (36\%), squamous carcinoma ( $22 \%$ ), not pathologically confirmed $(31 \%)$ and others, including large cell carcinoma $(11 \%)$ and carcinoid $(1.5 \%){ }^{4}$ In the current context of managing lung cancer and mesothelioma, molecular profiling has become of greater importance allowing more targeted therapies with potentially improved prognosis.

\section{Investigations}

Initial investigations for suspected lung cancer or mesothelioma include imaging in the form of a chest radiograph followed by computed tomography (CT) and, frequently, positron emission tomography (PET) to provide diagnostic and staging information. PET has limitations in the investigation of malignant mesothelioma and is not recommended in patients who have had prior talc pleurodesis, although it may have a role in detecting metastases. ${ }^{3}$ Current guidelines recommend the primary investigation should provide the most information for both diagnosis and staging, with least risk to the patient. ${ }^{1,3}$ Mediastinal and subcarinal lymph node staging forms an important aspect

\section{Key points}

Pulmonary and pleural malignancies remain common and the majority present at an advanced stage.

Mutation testing within the cancer cells allows for more specific treatment using new systemic agents including immunotherapy.

Indwelling pleural catheter and chest drain insertion with pleurodesis should be offered in management of malignant mesothelioma.

Lung cancer screening trials show reduced lung cancer mortality and a stage shift with earlier detection.

KEYWORDS: Lung cancer, mesothelioma - 
of management of lung cancer and can be undertaken noninvasively via CT and more frequently via PET, with the latter being more accurate with a sensitivity and specificity of $77.4 \%$ (95\% confidence interval (CI) 65.3-86.1) and 90.1\% (95\% CI 85.3-93.5). ${ }^{5}$ Patients considered for radical treatment ultimately require invasive sampling of the mediastinal lymph nodes. Surgical mediastinoscopy, which has low complications rates but requires general anaesthesia, has been considered as the gold standard with reported sensitivity of 86 to $93 \%$ and diagnostic accuracy of 93 to $96 \% .{ }^{6}$ Endobronchial ultrasound (EBUS) is a less invasive alternative, which allows ultrasound visualisation and a real time sampling of mediastinal lymph nodes with reported sensitivity of 0.90 (CI 0.84-0.96). ${ }^{7}$ Combined EBUS and endoscopic ultrasound (EUS) provide a diagnostic yield for mediastinal lymph node sampling as high as $97 \% .{ }^{8}$ Current guidelines suggest that surgical mediastinal lymph node staging should be considered in patients with negative EBUS/EUS sampling if clinical suspicion for nodal involvement is high and would affect treatment options, and has been shown to increase diagnostic sensitivity by $9 \%$.

\section{Early stage lung cancer and screening}

Current evidence suggests that only $15 \%$ of lung cancer cases are diagnosed at an early stage and even in these, patients the 5-year survival rate was 54\%. ${ }^{1}$ For patients with localised early stage lung cancer who are fit for surgery, a lobectomy should be offered as it provides better survival outcomes. ${ }^{1}$ For patients who are not fit for lobectomy, a sub-lobar resection or stereotactic ablative radiotherapy should be considered, offering survival benefits compared to conventional fractionated radiotherapy. ${ }^{1}$ The National Lung Screening Trial research findings showed that low dose helical CT screening, compared to chest radiography, could reduce lung cancer mortality by $20 \%$, supporting screening for early detection of lung cancer, which can then be treated with curative intent. ${ }^{9}$ A randomised controlled screening trial included approximately 16,000 people aged 50 to 74 years with smoking history and revealed a $26 \%$ reduction in lung cancer deaths for men over a 10 -year period. More impressively, the rate of lung cancer mortality in women was reduced by 61 to $39 \%$ at 8 to 10 years of follow-up..$^{10,11}$ The study reported that 27,000 screening $\mathrm{CT}$ resulted in a $0.9 \%$ lung cancer detection rate with the positive predictive value of $41 \%$. In the screening group, $50 \%$ of cancers were at an early stage and less than $10 \%$ at stage 4 , compared with only $10 \%$ early stage in the control arm where around half were diagnosed at stage 4 .

Early stage lung cancers frequently present as solitary peripheral lung lesions, which may be challenging for obtaining tissue for histological diagnosis. While flexible bronchoscopy is useful for biopsying proximal endo-bronchial lesions it has low diagnostic yield for peripheral lesions. Percutaneous trans-thoracic CT guided needle lung biopsy has accuracy sensitivity and specificity of over $90 \%$, but has recognised risks of pneumothorax of 0 to $61 \%$, with 3.3 to $15 \%$ of patients requiring intercostal chest drain insertion, and 5 to $16.9 \%$ risk of intrapulmonary haemorrhage. ${ }^{12}$ Alternatives include radial EBUS guided biopsy, with a reported diagnostic yield from peripheral lesions at between $63.3 \%$ and $84.4 \%{ }^{13}$ Another new technique gaining wider usage is navigational bronchoscopy during which flexible bronchoscopy is guided by virtual bronchoscopy using ultra-thin CT to sample peripheral lung lesions. ${ }^{14} \mathrm{~A}$ recent large prospective multicentre study, NAVIGATE, showed diagnostic yield of $73 \%$ in 1,053 procedures, with the low complication rates including $4.3 \%$ of pneumothorax and $2.9 \%$ of pneumothorax requiring chest drain insertion. ${ }^{15}$ There is no doubt that once national lung cancer screening is introduced these newer techniques will assume even greater importance. Meanwhile, a common strategy for peripheral lung nodules demonstrating fluorodeoxyglucose avidity on PET and no visible metastases is surgical resection with frozen section histology and immediate lobectomy if malignancy is confirmed.

\section{Developments in systemic therapies for lung cancer}

For SCLC chemotherapy in combination with (depending on the stage) radiotherapy to the lungs and cranial radiotherapy remain the main treatment options. NSCLC management has become more complex. In addition to conventional platinumbased chemotherapy new therapies have emerged, directed by the presence of different sensitising mutations within the cancer cells. For example, in patients with non-squamous (adenocarcinoma, large cell or undifferentiated) NSCLC who have sensitising mutations within the epidermal growth factor receptor gene (EGFR) to tyrosine kinase inhibitors (erlotinib, gefitinib or afatinib), treatment with these agents improves overall survival, progression free survival and quality of life, compared to platinum based chemotherapy. ${ }^{1}$ Similarly, in patients who have anaplastic lymphoma kinase (ALK) gene re-arrangement, present in 3 to $6 \%$ of NSCLC, crizotinib, which is an ALK inhibitor improved progression free survival (10.9 months) compared to platinumbased chemotherapy (7.0 months). ${ }^{1}$ The National Institute for Health and Care Excellence (NICE) consequently approved crizotinib and ceritinib for ALK positive advanced NSCLC. ${ }^{1}$ ROS-1 is a tyrosine kinase receptor related to ALK and its mutation is present in 1 to $2 \%$ of NSCLC. Crizotinib has also been approved by NICE for treatment of ROS-1 positive cancers. ${ }^{1}$ Another agent, osimertinib, can be used for locally advanced or metastatic disease in patients with cancer cells positive for the TT790 mutation of the gene coding for EGFR. There is also a role for a systemic treatment in patients with early stage of lung cancer including adjuvant chemotherapy after surgery. A different therapeutic option includes chemoradiotherapy, which may allow for access to durvalumab through an expanded access programme.

Immunotherapy including agents working through the immune check points such as programmed death 1 (PD-1) and programmed death ligand 1 (PD-L1), which are required protection of normal tissue from T-cell response, are used to manage patients with lung cancer or mesothelioma. ${ }^{16,17}$ PD-L1, which is a cell surface glycoprotein present on the activated antigen presenting cells and also on some lung cancer cells, normally binds to its receptor PD-1 resulting in the inhibition of activated T cells. Pembroluzimab is an IG 4 antibody targeting PD-1, which can be used as first line treatment for patients with squamous cell lung cancer with PD-L1 expression of more than $50 \%$ of cancer cells, having improved progression free survival significantly compared to conventional chemotherapy (10.3 vs 6 months). ${ }^{1}$ It is second line for patients who have $1-49 \%$ cells expression. Nivolumab, is a human immunoglobulin G4 immune checkpoint inhibitor, which binds to PD-1 and may be used in patients with no PD-L1 expression. ${ }^{1}$

\section{Management of mesothelioma}

Current guidelines suggest a limited role for surgery in the context of malignant mesothelioma. ${ }^{3}$ The MesoVATS study showed no 
difference between video assisted thoracoscopic surgery partial pleurectomy and medical pleurodesis. ${ }^{18}$ Current guidelines for the management of pleural effusion associated with malignant mesothelioma recommend pleurodesis using either talc slurry or thoracoscopic talc poudrage pleurodesis. ${ }^{3}$ In addition, patients can be offered indwelling pleural catheter insertion as first-line therapy, having been shown to have similar outcomes to talc pleurodesis. The MARS2 study exploring the effects of surgery is currently underway. The effects of systemic therapy are limited but may be offered to patients with good performance status. Cisplatin and pemetrexed, improved median overall survival (12.1 vs 9.3 months), with raltitrexed and cisplatin being an alternative regimen. ${ }^{19,20}$ The role of radiotherapy in the context of malignant mesothelioma is limited and the current guidelines do not recommend prophylactic radiotherapy to chest wall procedure tracts. ${ }^{3}$ There may also be a limited role for immunotherapy in managing malignant mesothelioma.

\section{Conclusions}

The investigations and management of pulmonary and pleural cancers has evolved in recent years. Technological improvements allow for more minimally invasive diagnostic procedures. Moreover, developments in systemic therapies are promoting individualised treatment options guided by different sensitising mutations within the cancer cells. This rapid, along with results from screening trials, are holding hope for better treatment outcomes for patients with pleural and lung cancers.

\section{References}

1 National Institute for Health and Care Excellence. Lung cancer: diagnosis and management. NICE guideline [NG122]. London: NICE, 2019.

2 Delgermaa V, Takahashi K, Park EK et al. Global mesothelioma deaths reported to the World Health Organization between 1994 and 2008. Bull World Health Organ 2011;89:716-24C.

3 Woolhouse I, Bishop L, Darlison L et al. British Thoracic Society Guideline for the investigation and management of malignant pleural mesothelioma. Thorax 2018;73:i1-30.

4 Royal College of Physicians. National Lung Cancer Audit. London: RCP, 2018. www.rcplondon.ac.uk/projects/national-lung-cancer-audit.

5 Schmidt-Hansen M, Baldwin DR, Hasler E et al. PET-CT for assessing mediastinal lymph node involvement in patients with suspected resectable non-small cell lung cancer. Cochrane Database Syst Rev 2014:CD009519.

6 Yasufuku K, Pierre A, Darling G et al. A prospective controlled trial of endobronchial ultrasound-guided transbronchial needle aspiration compared with mediastinoscopy for mediastinal lymph node staging of lung cancer. J Thorac Cardiovasc Surg 2011;142:1393-400.
7 Dong X, Qiu X, Liu Q, Jia J. Endobronchial ultrasound-guided transbronchial needle aspiration in the mediastinal staging of non-small cell lung cancer: a meta-analysis. Ann Thorac Surg 2013;96:1502-7.

8 Liberman M, Sampalis ], Duranceau A et al. Endosonographic mediastinal lymph node staging of lung cancer. Chest 2014:146:389-97.

9 Aberle DR, Adams AM, Berg CD et al. Reduced lung-cancer mortality with low-dose computed tomographic screening. NEJM 2011:365:395-409.

10 Yousaf-Khan U, van der Aalst C, de Jong PA et al. Final screening round of the NELSON lung cancer screening trial: the effect of a 2.5-year screening interval. Thorax 2017;72:48-56.

11 Field JK, Duffy SW. Lung cancer CT screening: are we ready to consider screening biennially in a subgroup of low-risk individuals? Thorax 2018;73:1006-7.

12 Manhire A, Charig M, Clelland C et al. Guidelines for radiologically guided lung biopsy. Thorax 2003;58:920-36.

13 Schuhmann M, Eberhardt R, Herth FJ. Endobronchial ultrasound for peripheral lesions: a review. Endosc Ultrasound 2013;2:3-6.

14 Asano F, Eberhardt R, Herth FJ. Virtual bronchoscopic navigation for peripheral pulmonary lesions. Respiration 2014;88:430-40.

15 Khandhar S], Bowling M, Flandes ] et al. Electromagnetic navigation bronchoscopy to access lung lesions in 1,000 subjects: first results of the prospective, multicenter NAVIGATE study. BMC Pulm Med 2017:17:59-68.

16 Philips GK, Atkins M. Therapeutic uses of anti-PD-1 and anti-PD-L1 antibodies. Int Immunol 2015;27:39-46.

17 Rotte A, Jin JY, Lemaire V. Mechanistic overview of immune checkpoints to support the rational design of their combinations in cancer immunotherapy. Ann Oncol 2018;29:71-83.

18 Rintoul RC, Ritchie AJ, Edwards JG et al. Efficacy and cost of videoassisted thoracoscopic partial pleurectomy versus talc pleurodesis in patients with malignant pleural mesothelioma (MesoVATS): an open-label, randomised, controlled trial. Lancet 2014;384:1118-27.

19 Vogelzang NJ, Rusthoven JJ, Symanowski J et al. Phase III study of pemetrexed in combination with cisplatin versus cisplatin alone in patients with malignant pleural mesothelioma. J Clin Oncology 2003:21:2636-44

20 van Meerbeeck JP, Gaafar R, Manegold C et al. Randomized phase III study of cisplatin with or without raltitrexed in patients with malignant pleural mesothelioma: an intergroup study of the European Organisation for Research and Treatment of Cancer Lung Cancer Group and the National Cancer Institute of Canada. J Clin Oncol 2005;23:6881-9.

Address for correspondence: Dr Jack A Kastelik, Department of Respiratory Medicine, Hull University Teaching Hospitals NHS Trust, University of Hull and Hull York Medical School, Castle Hill Hospital, Castle Road, Cottingham, East Yorkshire HU16 5JQ, UK.

Email: jack.kastelik@hey.nhs.uk 